Check for updates

Cite this: J. Mater. Chem. A, 2021, 9, 21835

Received 13th April 2021

Accepted 22nd August 2021

DOI: $10.1039 / \mathrm{d} 1 \mathrm{ta0} 3105 \mathrm{~h}$

rsc.li/materials-a

\section{Interfacial study of clathrates confined in reversed silica pores $\dagger$}

\author{
Paulo G. M. Mileo, (D) a Sven M. J. Rogge, (D) a Maarten Houlleberghs, (D) \\ Eric Breynaert, (iD ${ }^{b}$ Johan A. Martens (iD ${ }^{b}$ and Veronique Van Speybroeck (D) *a
}

\begin{abstract}
Storing methane in clathrates is one of the most promising alternatives for transporting natural gas (NG) as it offers similar gas densities to liquefied and compressed NG while offering lower safety risks. However, the practical use of clathrates is limited given the extremely low temperatures and high pressures necessary to form these structures. Therefore, it has been suggested to confine clathrates in nanoporous materials, as this can facilitate clathrate's formation conditions while preserving its $\mathrm{CH}_{4}$ volumetric storage. Yet, the choice of nanoporous materials to be employed as the clathrate growing platform is still rather arbitrary. Herein, we tackle this challenge in a systematic way by computationally exploring the stability of clathrates confined in alkyl-grafted silica materials with different pore sizes, ligand densities and ligand types. Based on our findings, we are able to propose key design criteria for nanoporous materials favoring the stability of a neighbouring clathrate phase, namely large pore sizes, high ligand densities, and smooth pore walls. We hope that the atomistic insight provided in this work will guide and facilitate the development of new nanomaterials designed to promote the formation of clathrates.
\end{abstract}

\section{Introduction}

In light of the growing environmental concerns in the last few decades, developing alternatives to minimize the carbon footprint is of utmost importance. Natural gas (NG) is one of the cleanest alternatives for energy generation. However, its widespread use is severely hampered by its high transportation costs, mainly due to its low density. Storing NG constituents as solidified natural gas (SNG) in the form of methane clathrates has been suggested as one of the most promising alternatives for NG transportation, mainly due to its environmentally friendly constituents and low safety risk. ${ }^{1}$ Clathrates are cagelike structures where water molecules surround usually smallsized molecules with highly directional hydrogen bonds. These materials typically present a well-defined crystallinity, represented by three main structures: I, II and $\mathrm{H}^{2}$ Methanecontaining clathrates usually form structure I (S-I) networks. ${ }^{3}$

The synthesis of methane clathrates even on a laboratory scale still poses crucial challenges that need to be tackled before this technology can become economically feasible. Especially, the low nucleation kinetics and high pressure required to initiate clathrate nucleation from bulk water are severe

${ }^{a}$ Center for Molecular Modeling (CMM), Ghent University, Technologiepark 46, B-9052 Zwijnaarde, Belgium. E-mail: veronique.vanspeybroeck@ugent.be

${ }^{b}$ Center for Surface Chemistry and Catalysis, Katholieke Universiteit Leuven, Celestijnenlaan 200F, 3001 Heverlee, Belgium

$\dagger$ Electronic supplementary information (ESI) available: Modelling and simulation strategies adopted for the reversed silica and clathrate microscopic models. See DOI: 10.1039/d1ta03105h problems increasing the production costs. ${ }^{4}$ Such conditions are usually relented by employing chemical additives (e.g. THF and surfactants) $)^{5,6}$ though this jeopardizes the $\mathrm{CH}_{4}$ volumetric storage in the hydrate. ${ }^{1,7}$ Alternatively, clathrate formation can be promoted under confinement in nanoporous materials. Under nanoconfinement, the $\mathrm{CH}_{4}$-water contact area is increased in comparison with bulk water, reducing the clathrate formation pressures compared to the bulk. ${ }^{4,8}$ Interestingly, storage in nanopores does not hamper the overall $\mathrm{CH}_{4}$ capacity and in some cases may even overcome the storage capacity observed in natural deposits. ${ }^{9}$

A fundamental understanding of the mechanisms involved in the promotion of clathrate formation through confinement in nanoporous environments is still rather limited due to the complex nature of this phenomenon. However, as pointed out in a recent review, ${ }^{10}$ mesoporous materials with moderate hydrophobicity are optimal candidates for promoting gas hydrate formation, as they allow a balance between (i) overcoming capillary forces, allowing the population of the pores, and (ii) preserving the preferential tetrahedral orientation of water molecules close to the pore's surface, an essential feature for the formation of clathrate cages. Besides their role in the hydrate formation, hydrophobic surfaces also seem to interact rather strongly with clathrate interfaces..$^{11,12}$ These fundamental considerations have been confirmed by recent studies, which revealed clathrates to be formed at faster rates and at milder temperatures than in bulk water when confined in porous materials displaying hydrophobic surfaces, such as activated carbon $^{9,13,14}$ and metal-organic frameworks. ${ }^{15-18}$ 
Silica matrices have also been widely studied ${ }^{19-21}$ as media for clathrate formation, mostly motivated by the need to understand how clathrates are formed in natural deposits. ${ }^{4}$ However, there seems to be some discrepancy in the literature on the role of silica surfaces in clathrate formation. While some experimental studies have revealed silica to act as an inhibitor of clathrate formation, ${ }^{\mathbf{2 0 , 2 2}}$ theoretical studies have pointed out that silica pores promote clathrate growth either due to the slower mobility of water molecules next to the silica surfaces ${ }^{23}$ or due to the role of these surfaces as a source of methane molecules. ${ }^{24}$ However, a point of agreement between experimental and theoretical studies is the fact that hydrophobic silica surfaces are more effective in clathrate promotion. ${ }^{25,26}$

In this sense, it seems an interesting approach to direct the formation of clathrates in nanoporous silica materials by grafting the silica surface with hydrophobic moieties, such as alkyl groups, at the $\mathrm{SiO}_{2}$ surface. This approach is employed in the synthesis of well-known amorphous reversed silica materials, widely used in chromatography separation. However, other materials such as ordered mesoporous silica, e.g. SBA-15, could also be easily functionalized to incorporate such hydrophobic groups at their surface. Indeed, multiple experimental studies have demonstrated the outstanding clathrate promoting effects of hydrophobized silica surfaces. ${ }^{27-30}$ Nevertheless, considering the diversity of types, distribution and concentration of surface ligands available to be introduced into $\mathrm{SiO}_{2}$ matrices, as well as the different pore sizes of the parent matrix, it is important to establish clear structure-property relationships to guide the choice of high-performance materials without having to rely on serendipity-based experiments.

Earlier computational evaluations of clathrate nucleation in nanoconfinement shed light on how the interfacial interactions play a fundamental role in the organization and stabilization of confined clathrate structures. In particular, it was observed that clathrate networks grow with the support of rather unorganized water molecule layers on hydrophilic silica surfaces. ${ }^{\mathbf{8 2 4 , 3 1}}$ It was also observed that the less hydrophilic such surfaces are, the more organized are the water molecules in the vicinities of the pore walls. This ultimately favours the clathrate growth. ${ }^{25}$ Moreover, the interactions of methane molecules with the pore wall seem to be also of importance by favouring the formation of half cages as observed next to the siloxane surface of kaolinites. $^{32}$

Considering the fundamental role of interfacial interactions between the clathrate and the pore walls in the structural stabilization of clathrate structures, we judge it necessary to obtain fundamental insight into the interfacial characteristics of different alkyl-grafted $\mathrm{SiO}_{2}$ surfaces. Therefore, we created $\mathrm{SiO}_{2}$ models in which various parameters such as the pore size, ligand density and ligand type are systematically varied to obtain insight into their role in the stability of clathrate structures. For each of the obtained structural models, we investigate the structural stability of the formed clathrate phase and examine whether the clathrate structure is retained when increasing the temperature above the equilibrium temperature.

In this work, we computationally studied the interfacial configurations of clathrate structures at the microscopic level by systematically changing the pore size of the silica matrix as well as the density and type of alkyl groups. Using an octylfunctionalized silica surface as a starting point, we first studied the impact of pore size and ligand density on the stability of clathrates. This was followed by a comparative study of the effect of different $\mathrm{SiO}_{2}$ ligands on the stability of adjacent clathrate phases. In Fig. 1, we summarize the main modelling strategy used in this work.

\section{Methods}

\section{Modelling strategy}

We started the construction of our reversed silica microscopic models by cleaving a bulk glass-like silica lattice along the $(0$ 0 1) plane and saturating uncoordinated $\mathrm{Si}$ and $\mathrm{O}$ atoms with $-\mathrm{OH}$ and $-\mathrm{H}$ atoms respectively. This procedure resulted in a 21 A-thick $60.15 \AA \times 60.15 \AA$ surface $\mathrm{SiO}_{2}$ slab containing a silanol density of $10.8 \mu \mathrm{mol} \mathrm{m} \mathrm{m}^{-2}$. Although this value is somewhat higher than the usual silanol surface concentration reported in silica $\left(8.0 \pm 1.0 \mu \mathrm{mol} \mathrm{m}{ }^{-2}\right),{ }^{33}$ such an extensive array of silanol groups facilitates the random placement of ligands in different densities. ${ }^{34}$

Some of these silanol groups were then replaced by monomeric ligands positioned orthogonally with respect to the $\mathrm{SiO}_{2}$ surface. Initially, dimethyloctylsilane (C8) was selected for an extensive investigation on the effect of different ligand concentrations and pore sizes on the interactions between reversed silica and clathrate surfaces. To perform this study, we generated two microscopic models with different C8 chain concentrations: one with $2.7 \mu \mathrm{mol} \mathrm{m} \mathrm{m}^{-2}$ and another with $5.6 \mu \mathrm{mol} \mathrm{m}{ }^{-2}$. Such C8 concentrations were respectively in agreement with lower (2-3.5

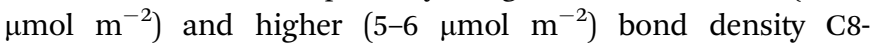
terminated silica. ${ }^{35}$ From these structural models, four different simulation cells were created by introducing two void zones $(25 \AA$ and $60 \AA$ ) perpendicular ( $z$ direction) to the C8-terminated $\mathrm{SiO}_{2}$ surfaces. Subsequently, $5 \times 5 \times 2$ and $5 \times 5 \times 5 \mathrm{CH}_{4}$ clathrate super cells constructed from an experimentally derived structure I clathrate unit cell obtained from the literature ${ }^{3}$ were cleaved along the $\left(\begin{array}{lll}0 & 0 & 1\end{array}\right)$ plane and introduced into the $25 \AA$ and $60 \AA$ void zones of the reversed silica models. For convenience, the resulting microscopic models, which presented either small (sp) or large pores (lp) and either a low (ld) or high density (hd) of C8 chains, were renamed $s p-h d, s p$-ld, $l p-h d$, and $l p$-ld, as shown in Fig. $1 \mathrm{~b}$ and $\mathrm{S} 1 . \dagger$

To study the effect of different alkyl groups on the interactions between the reversed silica and clathrate phases, the preceding structural model with a larger pore and a higher concentration of alkyl terminations $(l p-h d)$ was selected and later used as a template for the construction of two new models by replacing its terminal C8 ligands with either cyclopentyl (cC5) or trimethylsilane (C1) ligands. For simplicity, each of these models were respectively named $\mathrm{SiO}_{2}-\mathrm{C}_{8}, \mathrm{SiO}_{2}-\mathrm{cC}_{5}$, and $\mathrm{SiO}_{2}-\mathrm{C}_{1}$. A non-grafted (i.e. silanol terminated) $\mathrm{SiO}_{2}$ model $^{36}$ was also constructed as a reference for the hydrophobically grafted pores. Finally, a 60 Å void zone was created perpendicular to the reversed silica surfaces for the introduction of a $5 \times 5 \times 5 \mathrm{CH}_{4}$ clathrate super cell. A comparison between these different 

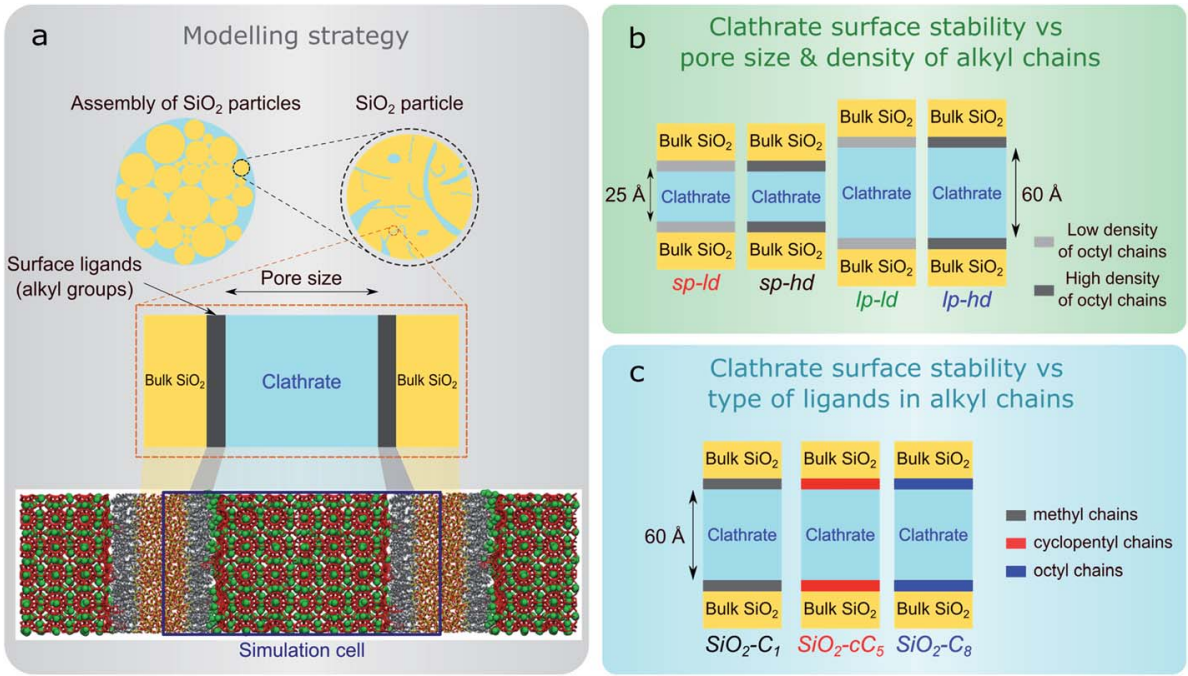

Fig. 1 Schematic figure displaying (a) the modelling of reversed silica pores using a planar representation, focused on the surface effects between the clathrate and the pore wall, (b) the four different structural models containing either smaller (sp) or larger pores (lp) and either a low (ld) or high (hd) density of ligands $(s p-l d, s p-h d, l p-l d$, and $l p-h d)$ to study the influence of the silica pore sizes and the density of alkyl chains on the stability of confined clathrate structures, and (c) the three models $\left(\mathrm{SiO}_{2}-\mathrm{C}_{1}, \mathrm{SiO}_{2}-\mathrm{cC}_{5}\right.$ and $\left.\mathrm{SiO}_{2}-\mathrm{C}_{8}\right)$ to study the clathrate stability considering different surface ligands.

structural models is displayed in Fig. 1c and S2. $\dagger$ In Table $\mathrm{S} 1 \dagger$ we report the volumetric methane capacities associated with each of these models.

Bonded interactions were taken into account by harmonic and cosine potentials and non-bonded interactions were described by Lennard-Jones (LJ) and coulombic potentials representing respectively the dispersive and electrostatic interactions. The bonded and non-bonded parameters attributed to the $\mathrm{SiO}_{2}$ atoms were taken from the literature, ${ }^{37}$ while the corresponding parameters assigned to the alkyl chains were obtained from the OPLS-AA model. ${ }^{38}$ Atomic point charges were obtained from single point energy calculations using the ChelpG method (see Fig. S3 $\dagger$ ) with the PBE functional ${ }^{39,40}$ and the 6-311g(d,p) basis set $^{41-43}$ as implemented in the Gaussian 16 software. ${ }^{44}$

Water molecules were described by the 4-site TIP4P/Ice model, which was designed to represent the experimental liquid-ice P-T diagram. ${ }^{45} \mathrm{CH}_{4}$ molecules are represented by the TRAPPE united atom model. ${ }^{46}$ The same combination of water and methane potentials was recently successfully employed for the study of clathrates' aggregation. ${ }^{8}$

\section{Molecular simulations}

Molecular dynamics calculations were carried out for all the aforementioned structural models using the LAMMPS software $^{47}$ in an isothermal-isobaric (NPT) ensemble using a NoseHoover ${ }^{\mathbf{4 8 , 4 9}}$ thermostat and barostat with respective temperature and pressure relaxation constants of $0.1 \mathrm{ps}$ and $1 \mathrm{ps}$. A timestep of 1 fs was used to solve Newton's equation of motion. Tail corrections were not used since they were reported ${ }^{8,50}$ to induce non-realistic clathrate behaviour. The systems were equilibrated for 500 ps during which the temperature was slowly increased from $100 \mathrm{~K}$ to $260 \mathrm{~K}$ at a pressure of $100 \mathrm{bar}$. Thereafter, 10 ns runs were carried out at 60 bar and $260 \mathrm{~K}$. Such temperature and pressure values are consistent with the range of moderate operating conditions employed in confined methane clathrates. ${ }^{\mathbf{8} 51}$ We extended this simulation time up to $100 \mathrm{~ns}$ for some selected systems and observed no structural changes, confirming that 10 ns was enough to achieve equilibration (see the ESI $\dagger$ ). Complementary calculations to test the stability of the clathrate structures out of their stable conditions were carried out at 60 bar and $300 \mathrm{~K}$. A spherical cut-off of $12 \AA$ was used to evaluate the dispersive interactions, whilst the electrostatic interactions were computed using a particleparticle particle-mesh (PPPM) solver ${ }^{52}$ with a $10^{-5}$ tolerance.

\section{Results and discussion}

\section{Impact of pore size and ligand concentration on the clathrate interface}

As a first step in our description of clathrate structures confined in reversed silica materials, we evaluated the effect of the silica pore size and the concentration of alkyl ligands on the interfacial clathrate organization by carrying out $10 \mathrm{~ns}$ force field based molecular dynamics (MD) calculations on the four structural models $(s p-h d, s p-l d, l p-h d$, and $l p-l d)$ created by introducing structure I (S-I) $\mathrm{CH}_{4}$ clathrate structures into either small ( $\sim 25 \AA$, sp) or large ( $\sim 60 \AA$, lp) pores of octyl-bonded silica slabs $\left(\mathrm{SiO}_{2}-\mathrm{C}_{8}\right)$ containing either a low (ld) or high (hd) density of alkyl chains (see Fig. 1b). Silica-based materials are characterized by a diversity of pore sizes, varying between a few to hundreds of nanometers. We decided to consider systems with relatively small pore ranges in order to maximize the surface effects on the confined clathrate phase. Meanwhile, the ld and hd configurations correspond to average ligand density values usually reported in low and high density alkyl-grafted silicas. ${ }^{35}$ 
a

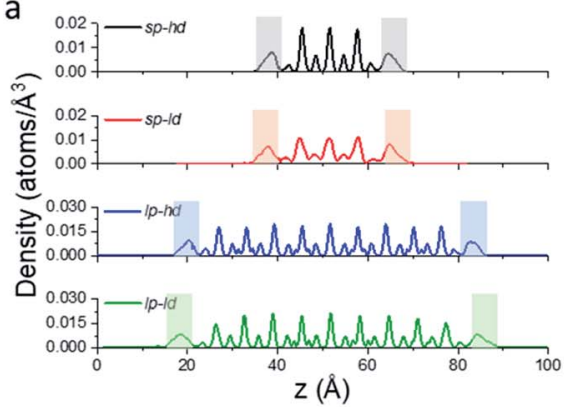

b

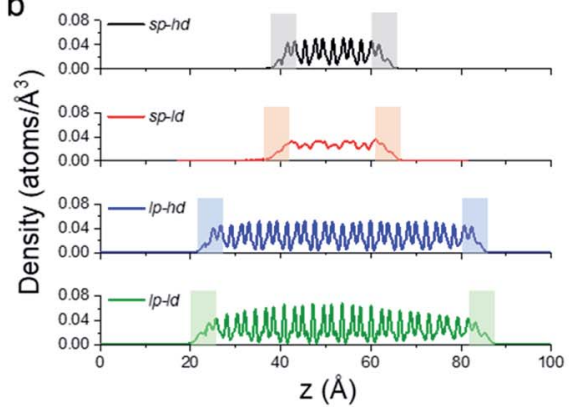

C
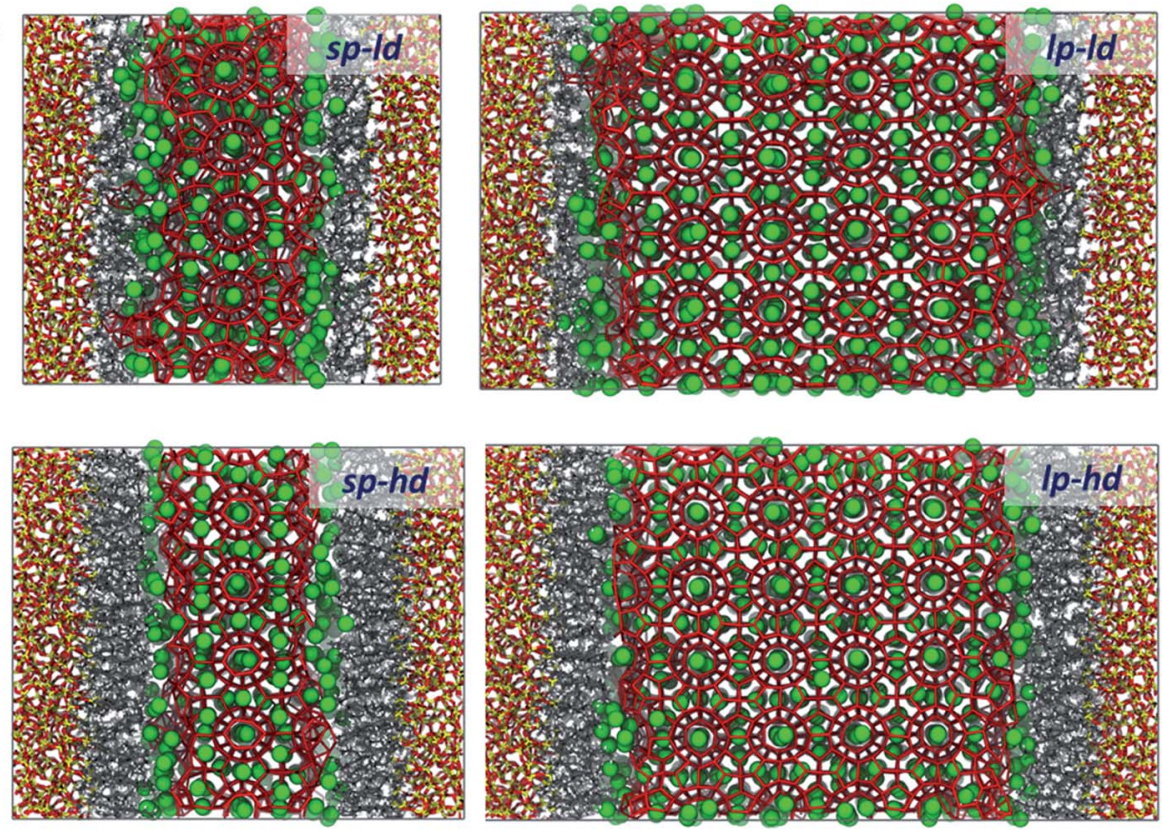

Fig. 2 Density of $\mathrm{CH}_{4}(\mathrm{a})$ and water molecules (b) along the axis orthogonal to the surface of the clathrate structures and the $s p$ - $h d$ (black line), $s p$-ld (red line), lp-hd (blue line), and $l p$-ld (green line) $\mathrm{SiO}_{2}-\mathrm{C}_{8}$ structural models. Shaded regions represent an $8 \AA$-wide interfacial zone on the clathrate phase next to the hydrophobic silica surfaces. Snapshots of each of these models after 10 ns long MD calculations showing how these organisations are observed in each model (c). $\mathrm{Si}, \mathrm{C}, \mathrm{H}$, and $\mathrm{O}$ elements and $\mathrm{CH}_{4}$ molecules are respectively shown in yellow, grey, white, red, and green. For clarity, water molecules are represented only by their oxygen atoms interconnected to each other with a $3.5 \AA$ cut-off distance.

Fig. 2a and $\mathrm{b}$ demonstrate the density of methane and water molecules in the clathrate phase nanoconfined in the four reversed silica models. Whereas their distribution shows periodic peaks in the bulk clathrate, the organisation of these molecules is considerably modified near the $\mathrm{SiO}_{2}-\mathrm{C}_{8}$ surface, where they assume a smoother distribution. This can be directly correlated with the local disruption of the hydrogen bonded clathrate networks due to the interactions of its individual components with the $\mathrm{SiO}_{2}-\mathrm{C}_{8}$ surface ( $c f$. Fig. 2c). In Fig. 2, it is also possible to notice that, compared to the water molecules, methane molecules are more delocalized in the vicinities of the octyl groups, because of their further penetration into the space delimited by the octyl chains.

In order to better track the effect of such modifications on the organization of the whole clathrate structure and the clathrate/wall interface, we compared the distribution of the orientational tetrahedral order (OTO) parameter ( $c f$. Fig. 3a and $\mathrm{ESI} \dagger$ ) of the water molecules in both regions. To this end, we defined the clathrate/ $\mathrm{SiO}_{2}-\mathrm{C}_{8}$ interfacial region as an $8 \AA$ wide zone extending from the water molecules closest to the pore's walls into the clathrate (see Fig. 3b). As observed in the shaded areas of Fig. $2 \mathrm{a}$ and b, $8 \AA$ corresponds to the furthest distance where we observed a relevant disruption of the water structure in all clathrate models. The remainder of the clathrate structure is defined as the inner clathrate region.

Fig. 3c and d show the extracted OTO distributions for the whole clathrate phase as well as for the inner and interfacial regions separately. For the total and inner clathrate regions (Fig. 3c), the OTO distribution is strongly peaked around 1. This is expected in an ideal S-I $\mathrm{CH}_{4}$ clathrate structure, as each water molecule is hydrogen bonded to four other water molecules. However, also a broader but smaller peak around an OTO value of 0.4 is present, typical of more disorganized systems, such as liquid water. In contrast, at the clathrate-wall interface (Fig. 3d), the OTO parameter assumes a bimodal distribution, which shows that some of the water molecules keep the tetrahedral environment observed in the bulk clathrate structure (i.e. with an OTO = 

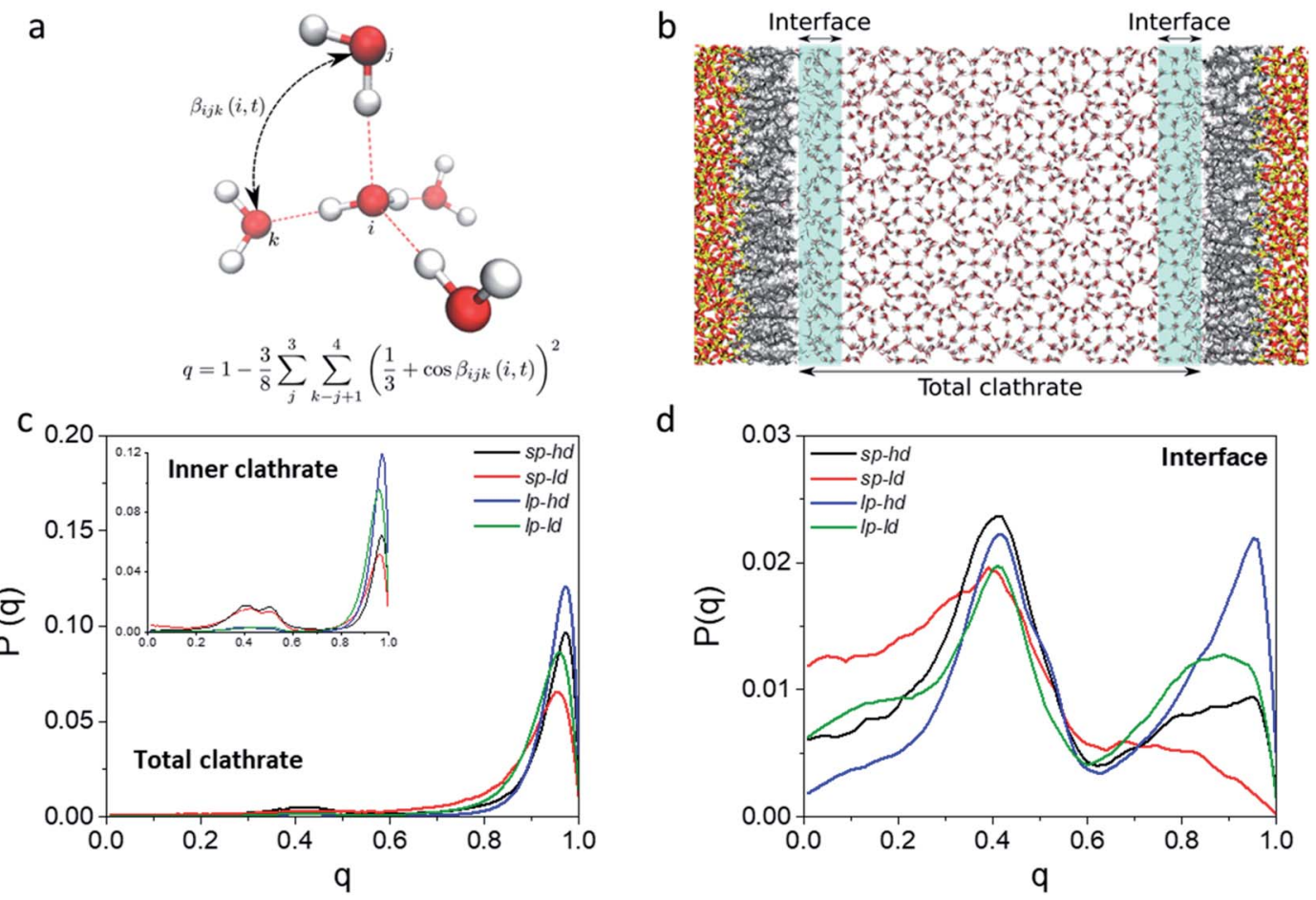

d

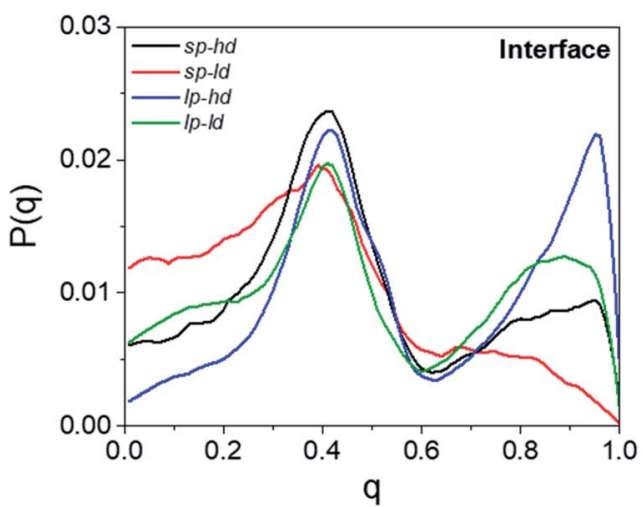

Fig. 3 (a) Representation of the orientational order parameter for water molecules, with $q$ values ranging from 0 for isolated molecules to 1 for a tetrahedrally hydrogen-bonded network. (b) Representation of a lp-hd structural model, defining the interfacial and the total clathrate regions. The inner clathrate region is defined as the total clathrate region without the interfacial regions. $\mathrm{CH}_{4}$ molecules were omitted for clarity. Probability distribution of the orientational tetrahedral order parameter obtained from the water molecules in the inner (inset) and whole clathrate (c) regions and at the clathrate $/ \mathrm{SiO}_{2}-\mathrm{C}_{8}$ interface (d).

1) and some form a liquid-like organization, with an OTO centred around 0.4.

Furthermore, from Fig. 3d we can observe that a higher proportion of molecules in the interfacial region assume a liquid-like configuration when smaller pores and lower alkyl densities are considered. While these effects are induced by the interface, they are also reflected in the overall organization of the inner clathrate phase (Fig. 3c), leading to an overall destabilization of the clathrate phase.

The aforementioned interfacial water disordering is reflected not only in the global ordering of the hydrogen bonded scaffold formed by the water molecules but also in their local environment. As one can see in the radial distribution functions (RDFs) and the corresponding cumulative distribution functions in Fig. S6, $\dagger$ the first solvation shell surrounding the water molecules is much more disorganized in the clathrate/pore wall interfacial region compared to the bulk clathrate. Consequently, each water molecule at this surface is, on average, surrounded by fewer neighbouring molecules. Instead of the typical arrangement of four other molecules surrounding each molecule observed at the core of the clathrate phase, in the $l p-h d$ interfacial region about three water molecules surround each $\mathrm{H}_{2} \mathrm{O}$ molecule while in the other configurations only about two $\mathrm{H}_{2} \mathrm{O}$ molecules form this first solvation shell.

From these observations, it seems that models displaying smaller pore sizes and a lower density of alkyl chains covering the $\mathrm{SiO}_{2}$ substrate perturb the clathrate phase more strongly. While the effect of pore size is a direct result of the proportionally larger interfacial region in small-pore structures, the effect of alkyl densities on the clathrate structure is not directly clear at this point. Therefore, to understand why lower alkyl surface concentrations favour the local disruption of the clathrate structure, we will take a closer look at the alkyl chains' configurations when present in either high or low density on the $\mathrm{SiO}_{2}$ surfaces. Two representative snapshots are shown in Fig. 4, which demonstrate that the octyl chains assume different configurations according to their relative concentration. At high density the alkyl chains keep their initial configurations orthogonal to the $\mathrm{SiO}_{2}$ matrix and extend into the clathrate phase ( $c f$. Fig. 4a), whereas at lower density they curl and tilt ( $c f$. Fig. $4 \mathrm{~b}$ and $\mathrm{S7} \dagger$ ) due to the high pressure exerted by the adjacent clathrate phase. In the low-density configuration, this pressure cannot be withstood in a configuration orthogonal to the surface due to the lack of mutual support and repulsion of neighbouring alkyl chains. This change in the alkyl chain configuration in ld configurations strongly disrupts the clathrate in the interfacial region due to (i) the shrinking of the alkyl zone atop the $\mathrm{SiO}_{2}$ matrix, as shown in Fig. $\mathrm{S} 8, \uparrow$ and (ii) the formation of void zones where both water and $\mathrm{CH}_{4}$ molecules can further penetrate ( $c f$. Fig. $4 \mathrm{~b}$ ).

Summarizing this first part on the influence of the pore size and the ligand density, we can conclude that large pores and high alkyl densities are beneficial to stabilize a well-structured clathrate phase. 

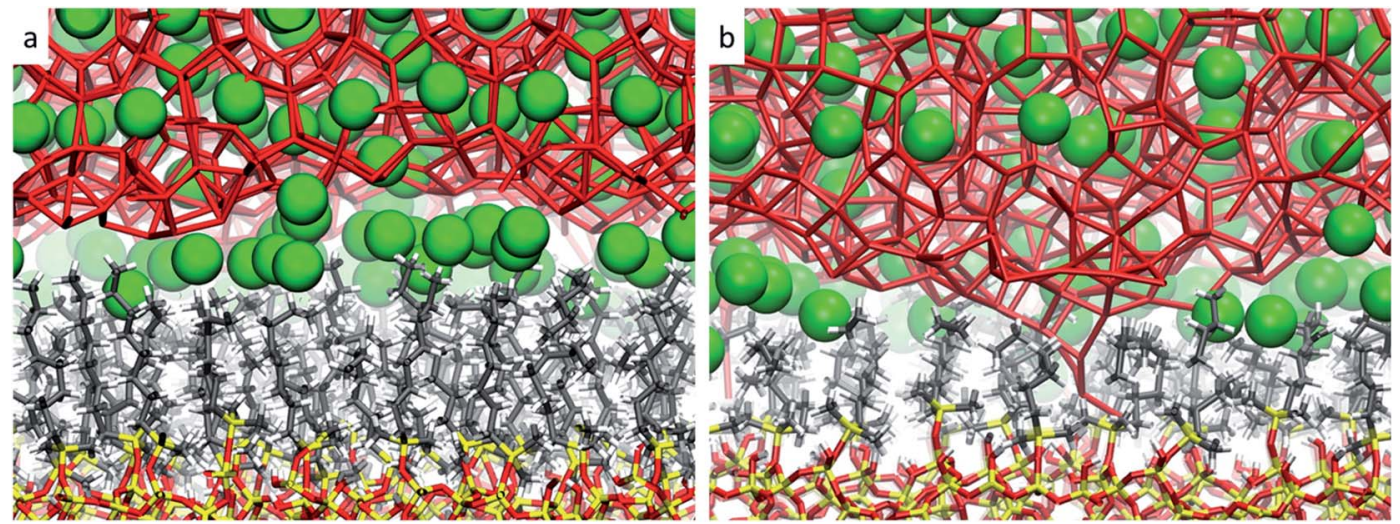

Fig. 4 Representative snapshots taken from the microscopic models $s p-h d(a)$ and $s p$-ld (b), displaying the configuration of the octyl chains when in respectively high or low densities over the $\mathrm{SiO}_{2}$ matrix. The same colour code as in Fig. 2 is used.

\section{Impact of ligand choice on local clathrate organization}

In light of the results obtained in the previous subsection, we will extend our discussion by evaluating the impact of different alkyl ligands on the water interfacial organization with high ligand density and large-pore configurations. In this sense, we constructed two other structural models, one containing trimethylsilane $\left(\mathrm{SiO}_{2}-\mathrm{C}_{1}\right)$ and another one containing cyclopentyl $\left(\mathrm{SiO}_{2}-\mathrm{CC}_{5}\right)$ as surface ligands. A similar analysis of the atomiclevel interactions in these models were explored using MD calculations (see Fig. 1c and computational details in the ESI $\dagger$ ).
As observed previously in the $l p-h d \mathrm{SiO}_{2}-\mathrm{C}_{8}$ model (henceforth simply called $\mathrm{SiO}_{2}-\mathrm{C}_{8}$ ), the distribution of the methane and water molecules in the interfacial regions of the newly constructed structural models ( $c f$. Fig. 5a and b) show a clear disruption compared to the bulk of the clathrate phase with the methane molecules being relatively less ordered in the vicinities of the organic ligands, consistent with what we reported in the previous sections. In order to further quantify the degree of interfacial water organization, we also compared the probability distribution of the orientational tetrahedral order parameter (Fig. 5c) in the interfacial

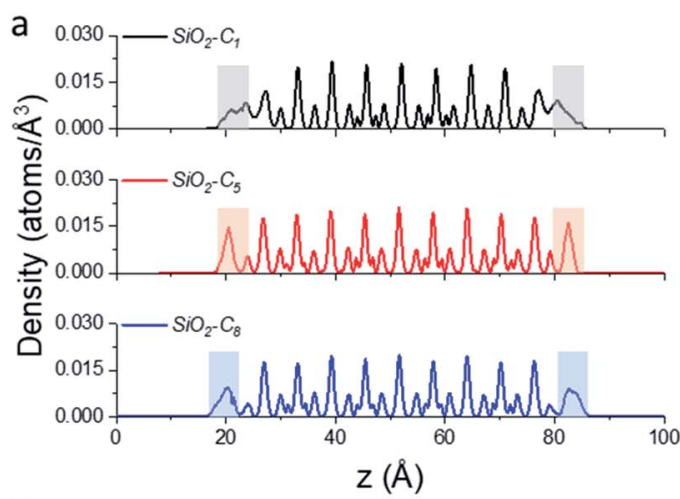

$\mathrm{C}$

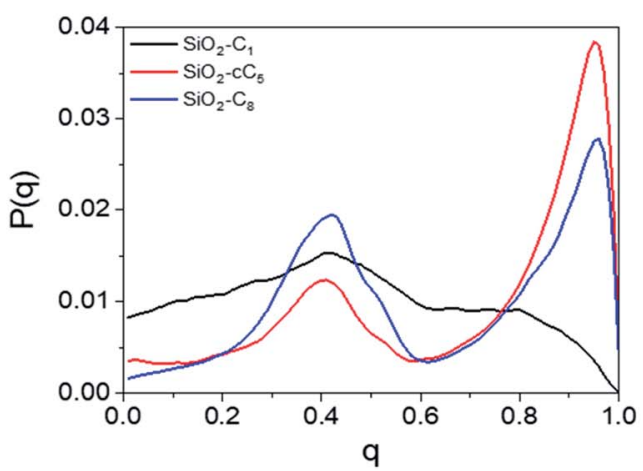

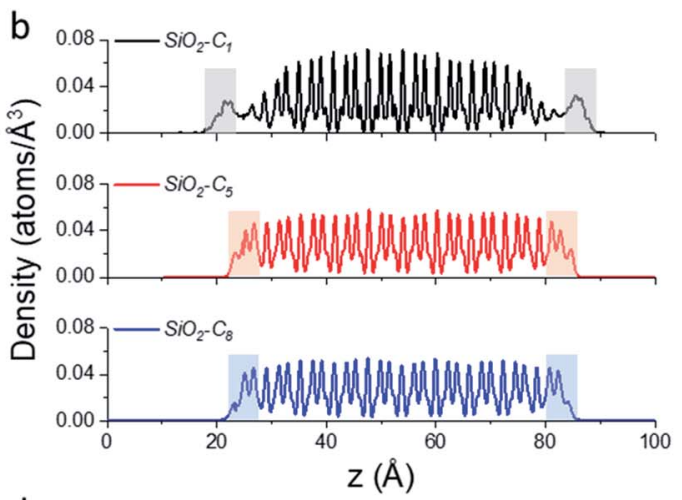

d

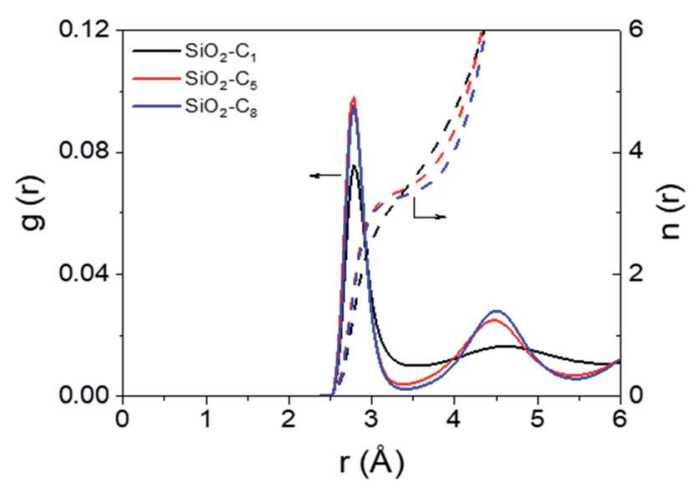

Fig. 5 Density of $\mathrm{CH}_{4}$ (a) and water (b) molecules along the axis orthogonal to the surface of the clathrate structures with their respective interfacial regions highlighted. Probability distribution of the orientational tetrahedral order parameter (c), and water donor-acceptor RDFs (solid line, left $Y$ axis) and their respective cumulative distribution functions (dashed lines, right $Y$ axis) (d) calculated at the clathrate/wall interface. SiO ${ }_{2}$ $\mathrm{C}_{1}, \mathrm{SiO}_{2}-\mathrm{CC}_{5}$, and $\mathrm{SiO}_{2}-\mathrm{C}_{8}$ models are respectively represented by black, red and blue lines. 
region. With respect to the OTO distribution observed in the $\mathrm{SiO}_{2}$ $\mathrm{C}_{8}$ model, the $\mathrm{SiO}_{2}-\mathrm{C}_{1}$ model is considerably less ordered, while the $\mathrm{SiO}_{2}-\mathrm{CC}_{5}$ model displays a similar OTO pattern, showing a higher degree of organization. This is also confirmed by the RDFs and their respective cumulative distribution function in Fig. 5d, which respectively show a less structured second solvation shell and a smaller number of neighbouring water molecules in the first solvation shell in the clathrate/wall interfacial region of the $\mathrm{SiO}_{2}-\mathrm{C}_{1}$ model. This is consistent with Raman spectroscopy measurements which showed a better water organization next to longer alkane chains at low temperatures. ${ }^{53}$ Fig. $5 \mathrm{c}$ also unveils a similar local organization of the interfacial water molecules in the $\mathrm{SiO}_{2}-\mathrm{cC}_{5}$ and $\mathrm{SiO}_{2}-\mathrm{C}_{8}$ structures.

However, while the $\mathrm{SiO}_{2}-\mathrm{cC}_{5}$ and $\mathrm{SiO}_{2}-\mathrm{C}_{8}$ models exhibit some similarity in terms of the water organisation pattern, the interactions between the alkyl ligands and the interfacial methane molecules are substantially different. The RDFs between the interfacial methane molecules and the carbon atoms of the alkyl chains $\left(\mathrm{C}_{\text {alkyl }}\right)$, shown in Fig. 6 a, indicate that the interactions between these species are considerably weaker in the $\mathrm{SiO}_{2}-\mathrm{C}_{8}$ model than in the $\mathrm{SiO}_{2}-\mathrm{cC}_{5}$ model. This is a very counterintuitive result, as one might imagine that the larger amount of carbon atoms in the octyl chains would imply stronger dispersive interactions with the methane molecules of the clathrate phase. Nevertheless, observing the surface contour plots of Fig. S9, $\dagger$ we can notice that the surface of the $\mathrm{SiO}_{2}-\mathrm{cC}_{5}$ slab is much less rugged than the $\mathrm{SiO}_{2}-\mathrm{C}_{8}$ surface. This
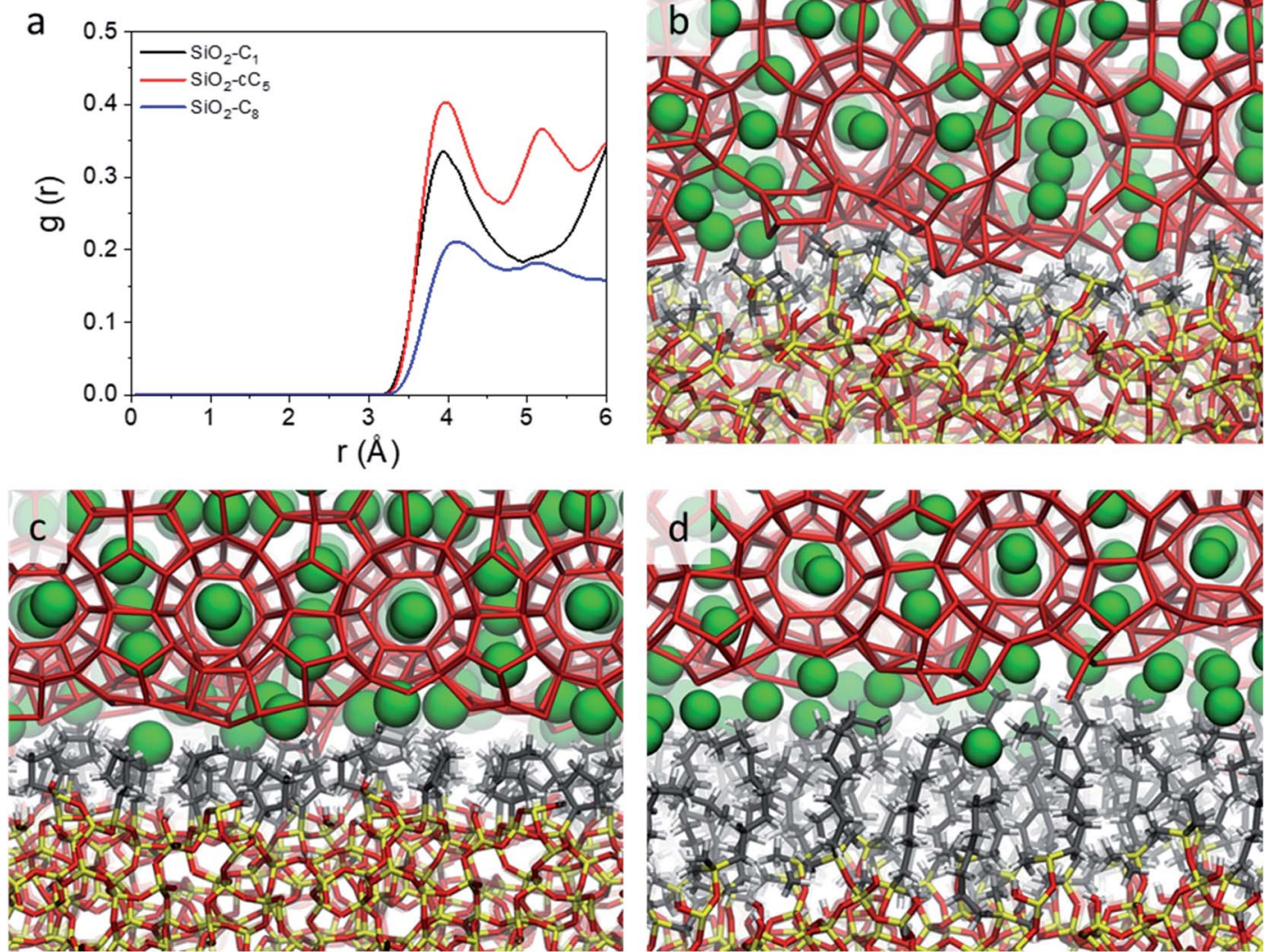

Fig. 6 Radial distribution functions representing the $\mathrm{CH}_{4}-\mathrm{C}_{\text {alkyl }}$ interactions (a) and representative snapshots displaying the organization of water and $\mathrm{CH}_{4}$ molecules at the interface of the clathrates in interaction with $\mathrm{C}_{1}$ (b), $\mathrm{cC}_{5}$ (c) and $\mathrm{C}_{8}$ (d) groups. The same colour code as in Fig. 2 is used. smoother cyclopentyl distribution would be the origin of the stronger interactions between this alkyl group and the $\mathrm{CH}_{4}$ molecules. Such superior performance of cyclopentyl groups in the stabilization of the confined clathrate phase is in line with recent experimental observations confirming the potential of cyclopentane as an efficient clathrate promoter molecule. ${ }^{54-56}$

As a consequence of the dissimilar surface roughness and alkyl- $\mathrm{CH}_{4}$ interaction patterns observed in $\mathrm{SiO}_{2}-\mathrm{C}_{8}$ and $\mathrm{SiO}_{2}-$ $\mathrm{cC}_{5}$, we observed a considerable difference in the $\mathrm{CH}_{4}$ distribution at the clathrate interface. In Fig. 6 , one can notice that the $\mathrm{CH}_{4}$ molecules close to the octyl surface are not as well organized as when they are in the vicinity of the cyclopentyl groups, with $\mathrm{CH}_{4}$ having more space to move on the interstices formed by the linear octyl chains. By measuring the mean square displacement of the methane molecules at the clathrate interfaces in contact with the $\mathrm{C}_{1}, \mathrm{cC}_{5}$ and $\mathrm{C}_{8}$ ligands (Fig. $\mathrm{S} 10 \dagger$ ), we confirmed this much higher mobility of the methane molecules close to the octyl surfaces.

From a practical viewpoint, this is an interesting result. Having a layer of rather mobile methane molecules at the clathrate-solid interface would imply faster $\mathrm{CH}_{4}$ recovery kinetics and also give more room to the introduction of other gases into the clathrate matrix, as recently suggested. ${ }^{57}$

\section{Comparison with ungrafted $\mathrm{SiO}_{2}$}

Having analysed the influence of different alkyl groups grafted onto the surfaces of $\mathrm{SiO}_{2}$ pores on the stability of a clathrate

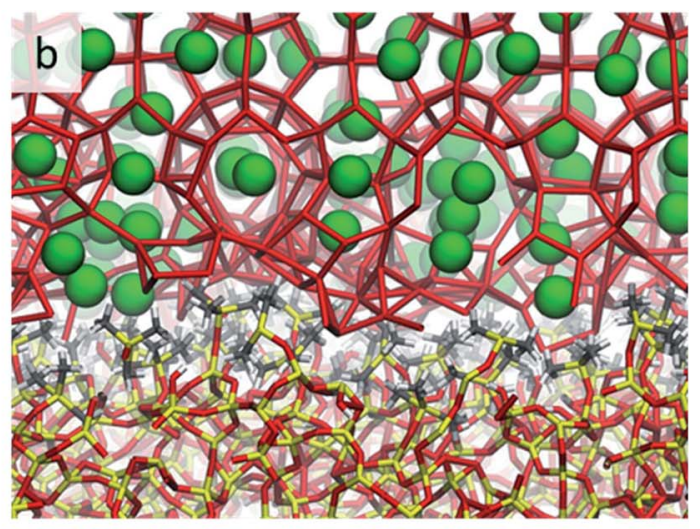


matrix, we decided to extend our investigation by studying how a pristine $\mathrm{SiO}_{2}$ surface compares to these hydrophobized silica models in terms of the stabilization of confined clathrate matrices.

For this purpose, we analyzed the water organization and $\mathrm{CH}_{4}$ mobility of the clathrate/pore wall interface in the best structural model in terms of clathrate stabilization, i.e. the $\mathrm{SiO}_{2}-\mathrm{cC}_{5}$ model, with those obtained in a typical $\mathrm{SiO}_{2}$ slit pore model ( $c f$. Fig. 7a). As seen in the integration of water donoracceptor $\left(\mathrm{O}_{\mathrm{w}}-\mathrm{O}_{\mathrm{w}}\right)$ RDF curves (Fig. 7b), there is a smaller degree of association among water molecules in the interfacial clathrate/pore wall region of the pristine $\mathrm{SiO}_{2}$ pore. Besides, as observed in the OTO plot (Fig. 7c), the clathrate matrix neighbouring an ungrafted $\mathrm{SiO}_{2}$ model displayed a larger proportion of molecules in a liquid-like organization, with an OTO value close to 0.4 . These results show that the clathrate scaffold is considerably better preserved next to a hydrophobic surface compared to the ungrafted structure. Such better preservation of the interfacial clathrate backbone is also reflected in a much smaller $\mathrm{CH}_{4}$ mobility predicted in the $\mathrm{SiO}_{2}$ $\mathrm{cC}_{5}$ model in comparison with the pristine $\mathrm{SiO}_{2}$ model (Fig. 7d).

This further preservation of the clathrate structure next to hydrophobic ligands is explained by the similarity of the hydrogen bond network around hydrophobic and amphiphilic groups (usually referred to as hydrophobic hydration) and the structure of hydrate groups, as confirmed both experimentally and computationally. ${ }^{58-61}$

Nevertheless, we observed that the interfacial water molecules next to the pristine $\mathrm{SiO}_{2}$ pore showed a higher degree of organization than the one observed in the $\mathrm{SiO}_{2}-\mathrm{C}_{1}$ model ( $c f$. Fig. 5c), which suggests that the clathrate-promoting effect of the hydrophobic hydration is very dependent on the alkyl chain length. Besides, the relative preservation of the clathrate scaffold next to the $\mathrm{SiO}_{2}$ surface shows that there is a relative interfacial compatibility of typical $\mathrm{SiO}_{2}$ surfaces and clathrates, as observed elsewhere. ${ }^{25,62}$

\section{Qualitative implications on clathrate metastability}

In the previous sections, we detailed the effects of different hydrophobic surfaces on the organization of the water molecules belonging to interfacial layers of confined clathrates under moderate operating conditions. We related this aspect to the inherent stability of the whole confined clathrate phase. Here, we will reinforce this link between stability and water surface organization by evaluating the preservation of clathrate phases in metastable states under unfavourable external conditions.

Bulk clathrates are known for their unexpected stability under conditions out of their structural equilibrium, the socalled "self-preservation" phenomenon. ${ }^{63,64}$ Similar to what is
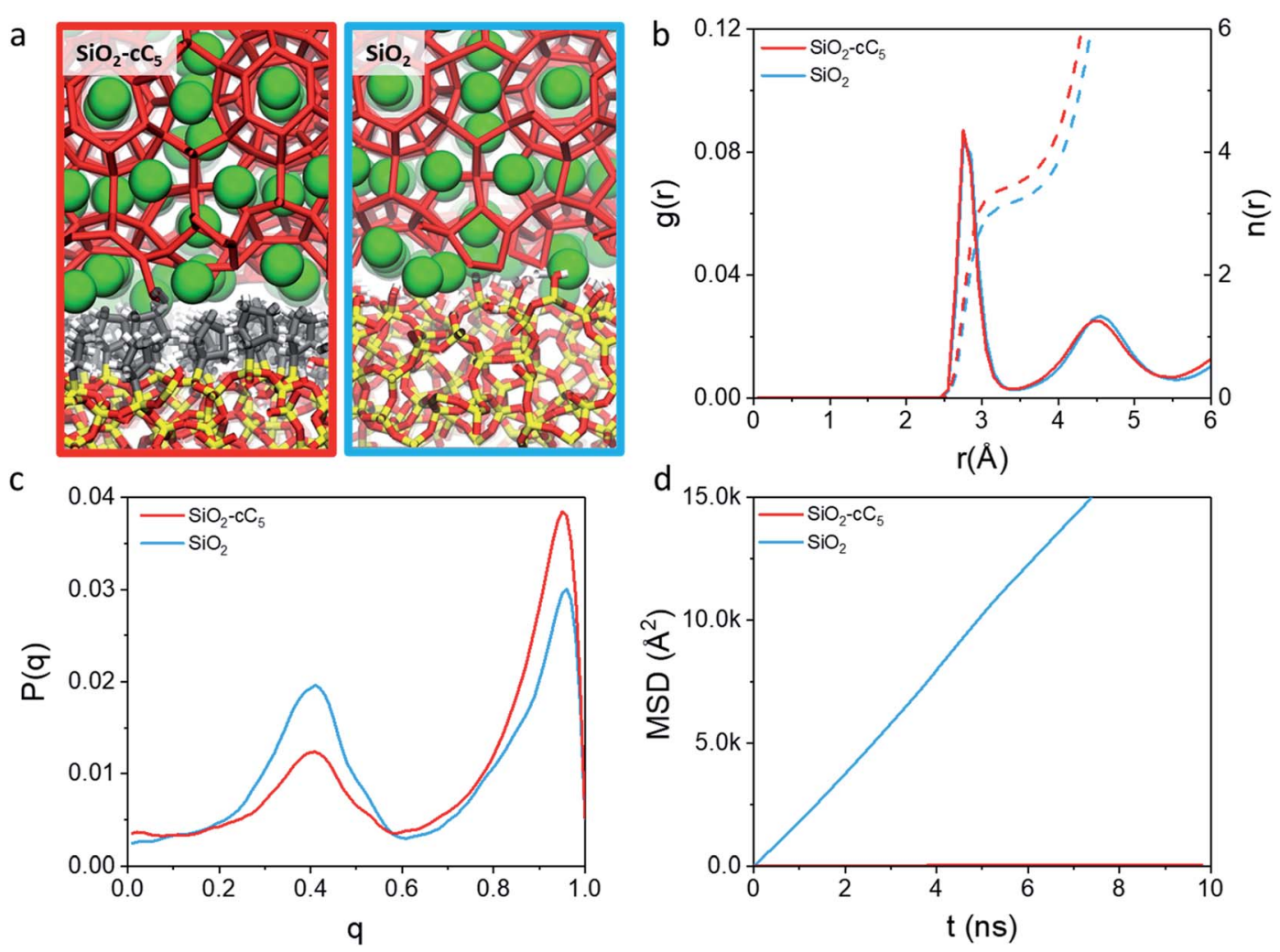

Fig. 7 Comparison between the interfacial clathrate/pore wall organization of the $\mathrm{SiO}_{2}-\mathrm{cC}_{5}$ and ungrafted $\mathrm{SiO}_{2}$ models, represented by a cropped snapshot (a), in terms of water donor-acceptor RDFs (solid lines, left $y$ axis) and their respective integration (dashed lines, right $y$ axis) (b) as well as the probability distribution of the orientational tetrahedral order parameter associated with them (c) and the mean squared displacement (MSD) of $\mathrm{CH}_{4}$ molecules calculated at the clathrate/pore wall interface (d). 
observed for the clathrate nucleation, confinement effects have shown to further potentialize this phenomenon. ${ }^{65}$

We decided to emulate such metastable clathrate configurations by carrying out $10 \mathrm{~ns}$ long MD calculations at 60 bar and $300 \mathrm{~K}$ using the different models listed in the previous sections. This allowed for a qualitative observation of the importance of pore size as well as the density and type of ligands for the preservation of clathrates out of their thermodynamic equilibrium.

Fig. S11 $\uparrow$ clearly shows a complete melting of the clathrate structure in smaller reversed silica pores regardless of the concentration of grafted ligands. Meanwhile, in larger pores, the clathrate bulk is observed to partially retain its configuration. Comparing the ld and hd configurations of the $\mathrm{SiO}_{2}-\mathrm{C}_{8}$ model, we can clearly notice in the former a larger disruption of the clathrate network, with a consequent higher concentration of $\mathrm{CH}_{4}$ molecules at the surface of the $\mathrm{C}_{8}$-grafted silica slab. This is observed on a much larger scale when methyl groups are used as grafting ligands, with the formation of methane bubbles next to the clathrate edges and the preservation of solely a thin clathrate layer at the center of the pore for the $\mathrm{SiO}_{2}-$ $\mathrm{C}_{1}$ model. In contrast, in the $\mathrm{SiO}_{2}-\mathrm{cC}_{5}$ model, the clathrate structure is completely preserved despite the unfavourable external temperature and pressure conditions.

From these observations, we notice a coincidence of the metastable clathrate stability here and the patterns of water interfacial organization discussed in the previous sections. This, along with our discussions on water organization and interfacial interactions between methane molecules and the ligands, suggests an unequivocal link between pore size/surface ligands and the clathrate stability.

\section{Conclusions}

In this work, we constructed molecular models to study the association of clathrate surfaces in reversed phase silica pores. By evaluating the water tetrahedral ordering and RDFs in the vicinities of the pore walls, we observed that pores containing larger pores and a higher density of hydrophobic ligands have a clearer clathrate stabilizing role. Moreover, we ascertained that different hydrophobic linkers may substantially alter the vicinal water microstructure and the $\mathrm{CH}_{4}$ organization. Therefore, ligands with a certain degree of rigidity and displaying a moderate chain length appeared to be more suitable for stabilizing clathrate surfaces. These results provide substantial insights for the experimental conception of new materials allowing the stabilization of clathrates at higher rates and milder temperatures.

\section{Conflicts of interest}

There are no conflicts to declare.

\section{Acknowledgements}

The authors acknowledge VLAIO for Moonshot funding (ARCLATH, No. HBC.2019.0110). S. M. J. R. acknowledges the
Fund for Scientific Research Flanders (FWO) for a junior postdoctoral fellowship (grant no. 12T3519N). M. H. and E. B. acknowledge FWO Flanders for an FWO-SB fellowship and a research grant $(1506118 \mathrm{~N})$ respectively. J. A. M. acknowledges the Flemish Government for long-term structural funding (Methusalem) and the European Research Council (ERC) for an Advanced Research Grant under the European Union's Horizon 2020 programme - Grant agreement No. 834134 (WATUSO). V. V. S. acknowledges the ERC for a Consolidator Research Grant under the European Union's Horizon 2020 programme - Grant agreement No. 647755 (DYNPOR) as well as the Research Board of Ghent University (BOF). The computational resources (Stevin Supercomputer Infrastructure) and services used in this work were provided by the VSC (Flemish Supercomputer Centre), funded by Ghent University, the Research Foundation - Flanders (FWO), and the Flemish Government - department EWI.

\section{Notes and references}

1 H. P. Veluswamy, A. Kumar, Y. Seo, J. D. Lee and P. Linga, Appl. Energy, 2018, 216, 262-285.

2 E. D. Sloan Jr, Nature, 2003, 426, 353-359.

3 F. Takeuchi, M. Hiratsuka, R. Ohmura, S. Alavi, A. K. Sum and K. Yasuoka, J. Chem. Phys., 2013, 138, 124504.

4 L. Borchardt, M. E. Casco and J. Silvestre-Albero, ChemPhysChem, 2018, 19, 1298-1314.

5 P. J. Herslund, K. Thomsen, J. Abildskov, N. von Solms, A. Galfré, P. Brântuas, M. Kwaterski and J.-M. Herri, Int. J. Greenhouse Gas Control, 2013, 17, 397-410.

6 U. Karaaslan and M. Parlaktuna, Energy Fuels, 2000, 14, 1103-1107.

$7 \mathrm{~J}$. Lee and J. W. Kenney III, Clathrate Hydrates, in Solidification, InTech, 2018.

8 K. Bin Yu and A. O. Yazaydin, J. Phys. Chem. C, 2020, 124, 11015-11022.

9 M. E. Casco, J. Silvestre-Albero, A. J. Ramírez-Cuesta, F. Rey, J. L. Jordá, A. Bansode, A. Urakawa, I. Peral, M. MartínezEscandell, K. Kaneko and F. Rodríguez-Reinoso, Nat. Commun., 2015, 6, 6432.

10 N. N. Nguyen, M. Galib and A. V. Nguyen, Energy Fuels, 2020, 34, 6751-6760.

11 A. A. Bertolazzo, P. M. Naullage, B. Peters and V. Molinero, J. Phys. Chem. Lett., 2018, 9, 3224-3231.

12 T. Head-Gordon, Proc. Natl. Acad. Sci. U. S. A., 1995, 92, 83088312.

13 L. Yan, G. Chen, W. Pang and J. Liu, J. Phys. Chem. B, 2005, 109, 6025-6030.

14 L. Borchardt, W. Nickel, M. Casco, I. Senkovska, V. Bon, D. Wallacher, N. Grimm, S. Krause and J. Silvestre-Albero, Phys. Chem. Chem. Phys., 2016, 18, 20607-20614.

15 C. Cuadrado-Collados, G. Mouchaham, L. Daemen, Y. Cheng, A. Ramirez-Cuesta, H. Aggarwal, A. Missyul, M. Eddaoudi, Y. Belmabkhout and J. Silvestre-Albero, $J$. Am. Chem. Soc., 2020, 142, 13391-13397.

16 D. Kim, Y.-H. Ahn and H. Lee, J. Chem. Eng. Data, 2015, 60, 2178-2185. 
17 L. Mu, B. Liu, H. Liu, Y. Yang, C. Sun and G. Chen, J. Mater. Chem., 2012, 22, 12246.

18 M. E. Casco, F. Rey, J. L. Jordá, S. Rudić, F. Fauth, M. Martínez-Escandell, F. Rodríguez-Reinoso, E. V. RamosFernández and J. Silvestre-Albero, Chem. Sci., 2016, 7, 3658-3666.

19 Z. Pan, Z. Liu, Z. Zhang, L. Shang and S. Ma, J. Nat. Gas Sci. Eng., 2018, 56, 266-280.

20 T. Uchida, T. Ebinuma and T. Ishizaki, J. Phys. Chem. B, 1999, 103, 3659-3662.

21 D.-L. Zhong, S.-Y. He, D.-J. Sun and C. Yang, Energy Procedia, 2014, 61, 1573-1576.

22 Y. P. Handa and D. Y. Stupin, J. Phys. Chem., 1992, 96, 85998603.

23 S. A. Bagherzadeh, P. Englezos, S. Alavi and J. A. Ripmeester, J. Phys. Chem. C, 2012, 116, 24907-24915.

24 S. Liang, D. Rozmanov and P. G. Kusalik, Phys. Chem. Chem. Phys., 2011, 13, 19856.

25 D. Bai, G. Chen, X. Zhang, A. K. Sum and W. Wang, Sci. Rep., 2015, 5, 12747.

26 S. Baek, J. Min, Y. Ahn, M. Cha and J. W. Lee, Energy Fuels, 2019, 33, 523-530.

27 Y. Guo, W. Xiao, W. Pu, J. Hu, J. Zhao and L. Zhang, Langmuir, 2018, 34, 10181-10186.

28 B. O. Carter, W. Wang, D. J. Adams and A. I. Cooper, Langmuir, 2010, 26, 3186-3193.

29 S. Fan, L. Yang, Y. Wang, X. Lang, Y. Wen and X. Lou, Chem. Eng. Sci., 2014, 106, 53-59.

30 W. Wang, C. L. Bray, D. J. Adams and A. I. Cooper, J. Am. Chem. Soc., 2008, 130, 11608-11609.

31 D. Bai, G. Chen, X. Zhang and W. Wang, Langmuir, 2011, 27, 5961-5967.

32 Y. Li, M. Chen, H. Song, P. Yuan, D. Liu, B. Zhang and H. Bu, Appl. Clay Sci., 2020, 186, 105439.

33 J. Nawrocki, J. Chromatogr. A, 1997, 779, 29-71.

34 K. A. Lippa, L. C. Sander and R. D. Mountain, Anal. Chem., 2005, 77, 7852-7861.

35 L. C. Sander, K. A. Lippa and S. A. Wise, Anal. Bioanal. Chem., 2005, 382, 646-668.

36 F. S. Emami, V. Puddu, R. J. Berry, V. Varshney, S. V. Patwardhan, C. C. Perry and H. Heinz, Chem. Mater., 2016, 28, 406-407.

37 C. D. Lorenz, E. B. Webb, M. J. Stevens, M. Chandross and G. S. Grest, Tribol. Lett., 2005, 19, 93-98.

38 W. L. Jorgensen, D. S. Maxwell and J. Tirado-Rives, J. Am. Chem. Soc., 1996, 118, 11225-11236.

39 J. P. Perdew, K. Burke and M. Ernzerhof, Phys. Rev. Lett., 1996, 77, 3865-3868.

40 J. P. Perdew, K. Burke and M. Ernzerhof, Phys. Rev. Lett., 1997, 78, 1396.

41 M. M. Francl, W. J. Pietro, W. J. Hehre, J. S. Binkley, M. S. Gordon, D. J. DeFrees and J. A. Pople, J. Chem. Phys., 1982, 77, 3654-3665.

42 R. Krishnan, J. S. Binkley, R. Seeger and J. A. Pople, J. Chem. Phys., 1980, 72, 650-654.

43 A. D. McLean and G. S. Chandler, J. Chem. Phys., 1980, 72, 5639-5648.
44 M. J. Frisch, G. W. Trucks, H. B. Schlegel, G. E. Scuseria, M. A. Robb, J. R. Cheeseman, G. Scalmani, V. Barone, G. A. Petersson, H. Nakatsuji, X. Li, M. Caricato, A. V Marenich, J. Bloino, B. G. Janesko, R. Gomperts, B. Mennucci, H. P. Hratchian, J. V Ortiz, A. F. Izmaylov, J. L. Sonnenberg, D. Williams-Young, F. Ding, F. Lipparini, F. Egidi, J. Goings, B. Peng, A. Petrone, T. Henderson, D. Ranasinghe, V. G. Zakrzewski, J. Gao, N. Rega, G. Zheng, W. Liang, M. Hada, M. Ehara, K. Toyota, R. Fukuda, J. Hasegawa, M. Ishida, T. Nakajima, Y. Honda, O. Kitao, H. Nakai, T. Vreven, K. Throssell, J. A. Montgomery Jr, J. E. Peralta, F. Ogliaro, M. J. Bearpark, J. J. Heyd, E. N. Brothers, K. N. Kudin, V. N. Staroverov, T. A. Keith, R. Kobayashi, J. Normand, K. Raghavachari, A. P. Rendell, J. C. Burant, S. S. Iyengar, J. Tomasi, M. Cossi, J. M. Millam, M. Klene, C. Adamo, R. Cammi, J. W. Ochterski, R. L. Martin, K. Morokuma, O. Farkas, J. B. Foresman and D. J. Fox, Gaussian Inc., Wallingford CT, 2016.

45 J. L. F. Abascal, E. Sanz, R. García Fernández and C. Vega, J. Chem. Phys., 2005, 122, 234511.

46 M. G. Martin and J. I. Siepmann, J. Phys. Chem. B, 1998, 102, 2569-2577.

47 S. Plimpton, J. Comput. Phys., 1995, 117, 1-19.

48 W. G. Hoover, Phys. Rev. A, 1986, 34, 2499-2500.

49 S. Nosé, J. Chem. Phys., 1984, 81, 511-519.

50 V. K. Michalis, J. Costandy, I. N. Tsimpanogiannis, A. K. Stubos and I. G. Economou, J. Chem. Phys., 2015, 142, 044501.

51 P. S. R. Prasad, B. S. Kiran and K. Sowjanya, RSC Adv., 2020, 10, 17795-17804.

52 R. W. Hockney and J. W. Eastwood, Computer Simulation Using Particles, IOP Publishing Ltd, 1st edn, 1988.

53 J. G. Davis, K. P. Gierszal, P. Wang and D. Ben-Amotz, Nature, 2012, 491, 582-585.

54 J. Zhang, P. Yedlapalli and J. W. Lee, Chem. Eng. Sci., 2009, 64, 4732-4736.

55 L. C. Ho, P. Babu, R. Kumar and P. Linga, Energy, 2013, 63, 252-259.

56 J. Zheng, B.-Y. Zhang, Q. Wu and P. Linga, ACS Sustainable Chem. Eng., 2018, 6, 11913-11921.

57 M. Cha, K. Shin, H. Lee, I. L. Moudrakovski, J. A. Ripmeester and Y. Seo, Environ. Sci. Technol., 2015, 49, 1964-1971.

58 F. Jiménez-Ángeles and A. Firoozabadi, ACS Cent. Sci., 2018, 4, 820-831.

59 N. Galamba, J. Phys. Chem. B, 2013, 117, 2153-2159.

60 N. Galamba, J. Phys. Chem. B, 2014, 118, 4169-4176.

61 J. Grdadolnik, F. Merzel and F. Avbelj, Proc. Natl. Acad. Sci. U. S. A., 2017, 114, 322-327.

62 Z. He, P. Linga and J. Jiang, Langmuir, 2017, 33, 1195611967.

63 C. Giavarini and F. Maccioni, Ind. Eng. Chem. Res., 2004, 43, 6616-6621.

64 L. A. Stern, S. Circone, S. H. Kirby and W. B. Durham, J. Phys. Chem. B, 2001, 105, 1756-1762.

65 S. Y. Misyura, Sci. Rep., 2016, 6, 30324. 